I wish to thank Mrs. G. A. Hill and Mr. J. Ratcliffe for experimental assistance.

R.A.F. Institute of Aviation Medicine,

P. SMTTH

Farnborough, Hants. Oct. 8.

${ }^{1}$ Smith, P., and Bennett, A. M. H., Nature, 181, 709 (1958).

${ }^{2}$ Shaw, K. N. F., and Trevarthen, J., Nature, 182, 797 (1958).

Hill, G. A., Ratcliffe, J., and Srnith, P., Nature, 182, 1160 (1958)

Both A. Jones, F. T., and DeEds, F., J. Biol. Chem. N29, Emerson, 0 .

s Armstrong, M. D., and McMillan, A., Fed. Proc., 16, 146 (1957).

${ }^{5}$ Armstrong, M. D., McMillan, A., and Shaw, K. N. F., Biochim. Biophys. Acta, 25, 422 (1957)

i Katwamoto, J., Chem. Abst., 48, 7727 (1954).

\section{Local Reaction to Non-metastasizing and Metastasizing Transplantable Tumours}

THE mechanism by which tumours spread into the surrounding tissues or produce metastases in distant organs is still an obscure problem. The reaction of the tissues of the host in relation to the rate of growth and metastasization has not been sufficiently investigated.

Bottiglioni and Prodi ${ }^{1}$ observed that only the stroma of metastasizing tumours is rich in a metachromatic substance.

The present investigation aimed at studying the local reaction to non-metastasizing and metastasizing transplantable tumours, namely, the Walker adenocarcinoma and a rat sarcoma (non-metastasizing tumours at expansive growth), rat tumour Guérin T58 (non-metastasizing, in our strain, at infiltrative growth), rat tumour Guérin $T 8$ (metastasizing, lymphotrope) and the rabbit Brown Pearce tumour (which produces metastases in lymph nodes and parenchymatous organs).

The tumours were transplanted with the usual technique, those of the rat subcutaneously, and the Brown Pearce tumour in the testis. The animals were killed when tumours had attained maximal development. Both primary tumours and metastases, when present, were removed with their surrounding tissue and studied with appropriative connective tissue stainings.

Investigations were carried out on material from sixty-five animals.

Around the grafts of Walker adenocarcinoma and rat sarcoma no connective tissue reaction was macroscopically detected; microscopically, the boundaries between tumour cells and host tissue are well outlined. The host tissue shows a considerable number of mastcells, forming a sort of barrier around the tumour mass. Some of these cells are degranulated, some packed with granules. The fibroblastic reaction is rare.

Tumour T58 seems to become identified with the adjacent tissues. Its cells penetrate into the dermis and muscle, directly replacing the tissues without any kind of reaction.

Around tumour $T 8$, in both the primary site and particularly around the metastases in superficial lymph nodes, a layer of loose connective tissue, sometimes of a mucous type, is observed; this tissue is rich in fibroblasts, and the loose fibrillar texture assumes a clear-cut metachromatic colour when stained with toluidine blue. Here and there the tissue takes the aspect of a thin extracellular network which shows intense metachromasia. If sections are pretreated with hyaluronidase, metachromasia does not occur. The mast-cells are scanty. Many round-shaped structures, which apparently are hectasic lymphatic vessels, are observed. The con- nective tissue is infiltrated with tumour cells which occasionally are also seen inside the lumen of these vessel-like structures.

Both around the primary tumour and the metastases (in the liver, kidney, lung, lymph nodes and peritoneal membranes) of Brown Pearce tumour an intense connective tissue reaction is observed with a large amount of highly metachromatic substance, which is destroyed by pretreatment with hyaluronidase. The tissue is of a loose fibrillar type, with often numerous fibroblasts and infiltrated with tumour cells.

The presence of a mast-cell barrier around the grafts of Walker adenocarcinoma and rat sarcoma which do not metastasize and grow extensively may indicate a defence against infiltration : such a hypothesis has already been suggested by Sylvén in relation to skin carcinogenesis ${ }^{2}$. This observation seems to be confirmed by the fact that there is little or no such reaction around tumour T58, which is infiltrative.

As regards metastasizing tumours, the tissue reaction is represented by a loose connective tissue which does not change into thickly fibrous tissue. This, with some differences, is a common character of the Brown Pearce and the T8 tumours. The reaction of connective tissue interposed between host and tumour tissue forms a sort of third section which is easily invaded by tumour cells and which, being rich in vascular formations (which are particularly evident around $T 8$ tumour), facilitates their passage into the circulation and consequently the onset of metastases.

These considerations suggest that the rate of growth as well as the ability to induce metastases may not be directly dependent upon intrinsic characters of the cell but rather upon the reaction induced and established by the tumour cell in the host tissue.

Institute of General Pathology,

C. Malmoni

University of Bologna.

${ }^{1}$ Bottiglioni, E., and Prodi, G., Lo Sperimentaie, 107, 150 (1957).

'Sylvén, B., Acta Unio contra cancrum, 10, 2 (1954).

\section{Changes in the Potassium Concentration and Metabolism of Red Blood Cells of the Lamb}

MaNy investigations have been made attempting to relate the potassium content and transport in red blood cells to the glycolytic activity of the cells ${ }^{1,2}$. Such investigations have utilized red blood cell suspensions which have been stored at low temperatures in order to obtain partial depletion of the cells with respect to potassium.

Widdas ${ }^{3}$ has shown that the potassium content of fotel sheep red blood cells is considerably higher than that found in the mature animals of the same breed. At birth the potassium concentration in the cells is high and decreases with increasing age of lamb. Thus successive samples of red blood cells from the same lambs can produce systems in which marked differences in the concentration gradient $\left(K^{+}\right)_{i} /\left(K^{+}\right)_{0}$ exist.

In preliminary experiments, we have made measurements of the potassium and sodium content, oxygen uptake and glycolytic activity of red cells obtained from lambs over the period 12-60 days of age.

Venous blood samples were taken into heparin, centrifuged, the red blood cells isolated, weshed with 